\title{
Virtual Reality Inspired Drugs (VRID): the Future Arena of Drug Discovery
}

\author{
Yaki Setty $^{1}$ (D) \\ Accepted: 22 July 2019/Published online: 8 August 2019 \\ (C) The Author(s) 2019
}

\begin{abstract}
The recent advancements of virtual reality (VR) technologies have paved the way for exciting new avenues in medical research. To date, VR technologies have been used as a training tool for behavioral interventions and for clinical diagnoses. I argue that VR technologies can serve to increase our understanding of disease progression and act as a means to design and test new treatments. To illustrate how virtual reality can aid in the development of drug delivery, I describe eBrain, a virtual reality platform that models drug administration to the brain. eBrain helps to study drug diffusion, uptake, and efficacy in silico. Also, eBrain has identified optimal treatments from a multitude of treatment protocols. I suggest to term the use of virtual reality technologies in the field of drug discovery VRID for Virtual Reality Inspired Drugs. The meaning of the word "VRID" in the Hebrew language is a "vein", highlighting the potential of the VRID concept to become a vein for novel drug discoveries. eBrain thus serves as a proof of concept for the VRID doctrine and its potential to revolutionize the drug design industry in the long term.
\end{abstract}

Keywords Simulations $\cdot$ Virtual reality $\cdot$ Drug development $\cdot$ In silico medicine $\cdot$ Computational medicine

Virtual reality technologies aim to simulate the physical presence in an imaginary computational space. These technologies are being rapidly developed and have been a focus in the video game industry. Often, VR is used to mimic a hypothetical reality in which users confront scenarios driven by interactions between them and the environment. One example is the video game series "Unreal," a first-person shooter video game (www.epicgames. com), which is centered around weapon-based combat in which the player controls a single character on a battlefield. VR is also extensively utilized in the aerospace industry and the military. In these fields, it is used for training and education purposes, such as training with flight simulators that mimic the experience in a virtual world. In some cases, training centers incorporate such simulations as a mandatory part of their curriculum $[1,2]$.

VR in medical and healthcare research is gaining increased attention, especially in the areas of imaging, training, and diagnostic tools. In the field of imaging, VR technologies have been used to improve performance and user experience in

This article is part of the Topical Collection on Medicine

Yaki Setty

yaki.setty@gmail.com

1 Gateway Institute for Brain Research, 3321 College Avenue, Fort Lauderdale, FL 33314, USA the correction of MRI segmentation errors [3], by the way of VR-based software that interactively guides the user. The VR-aided tool provided a more efficient, intuitive, and engaging alternative to the existing methods in the field. In surgical training, VR-based simulations have been incorporated in several programs as part of their stem education. To improve surgical planning skills, novices are trained on VR simulations that mimic the surgical procedure and receive feedback on their performance $[1,4-6]$. As alternative treatment and diagnosis methods, VR has been employed to expose patients to relevant virtual environments. This has been successfully utilized for stress release and pain reduction for patients suffering from chronic pain. Also, VR has been used to help rehabilitate motor function in patients with motor impairments [7]. In this case, VR simulation instructs patients in navigation scenarios with multimodal stimuli and provides feedback to better achieve the motor task-driven goals. The researchers reported an increase of $10 \%$ in performance for patients using the system. In mental health disorders, VR was used for exposure therapy for anxiety, depression, or phobias (in some cases by mimicking visual effects caused by LSD). VR simulations are used to monitor, diagnose, and aid the patient in an alternative virtual world created to address their condition [8]. Similarly, VR technologies has been employed for gait training in subacute stroke survivors [9] and in patients 
who suffer from central nervous system disorders [10]. The VR simulations give stroke patients a platform to practice physical activities and motor function and were found to improve various functional parameters (e.g., increase walking speed by over than 25\%) [9-11].

VR can be further utilized to accelerate drug development by providing an innovative technological framework in which to develop ideas for highly engineered drug products [12-14] (Fig. 1). Developing VR simulations from known experimental knowledge can enhance the process of reverse engineering, a biological system that is necessary for gaining insight into its function. This task requires exploration of biological-driven mechanisms that explain observations and concur with the current state of the field of the research in question. The VR simulation development process, on its own, serves as a gap analysis to delve deeper into areas that are not yet fully understood. This development forces formulation of a system-level understanding of the particular system being modeled. This process highlights interactions between parallel mechanisms and suggests new targets for drug development. Once completed, VR simulations can serve as a hypothesis generator. The simulation serves as a platform to study functionality and better understand the underlying mechanisms. Furthermore, potential drugs and their physicochemical properties can be tested to predict interactions between drugs and their target pathways. VR simulations are excellent platforms for drug repurposing analyses by testing their potential application on the target mechanisms. Ultimately, VR simulations can help in the course of designing new treatments and shortening the drug discovery period. Specifically, VR simulations can accelerate target identifications at the preclinical research stage, by providing a theoretical framework to generate novel ideas by scanning over multiple potential drugs and their impact on relevant pathways, mechanisms, and proteins. VR simulations can thus assist to explore system dynamics, test strategies, what-if scenarios, refute unsound hypothesis, and guide the design of novel experiments.

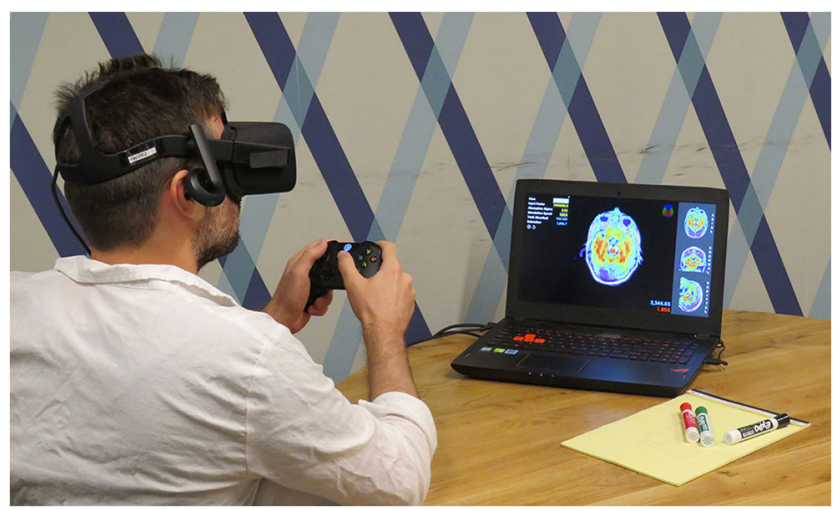

Fig. 1 Futuristic drug development: using eBrain with Virtual Reality goggles to develop treatment protocols
VR simulations add a novel layer to the pharmacodynamics of drugs by analyzing their effect on the simulated theoretical system in a three-dimensional space. The simulation can be constructed by data from any subject, allowing the simulation to mimic precise conditions and study specific scenarios for every individual patient. VR provides the means to study the pharmacokinetics of drugs by testing the drug absorption, distribution, metabolism, and excretion, via different delivery systems. VR simulations serve as an alternative and/or additional layer for the mathematical-based pharmacokinetic/pharmacodynamic (PK/PD) models that guide predictions of drug treatment strategy, drug concentration, and drug efficacy [15]. Drug testing using VR simulations can accelerate the screening of new drugs and provide information for Investigational New Drug (IND) applications [16]. VR simulations can provide new possible indications, suggest changes in the route of administration, and indicate the optimal dosage level. Additionally, VR can aid in the stratification of patient populations for clinical trials, based on genetic mutations, data classification, symptoms, or any other specified criterion. Furthermore, VR simulations may shorten the lead generation and screening phases in preclinical work, by theoretically testing therapeutic efficacy and predicting pharmacological and biological activities of lead compounds. VR simulations could allow the tuning of parameters such as potency, selectivity, or pharmacokinetics in order to gain a better understanding of the system. Newly found results and outputs from biological and clinical trials can be easily incorporated into the simulation in an iterative process to ensure that they concur with the most cutting edge results in a field of interest. Furthermore, VR simulations can mimic the function of multiple delivery systems. Thus, they can analyze the activity of a drug using different delivery systems and screen the efficacy of treatments. Moreover, VR simulations have the potential to go beyond the "one-size-fits-all" principle in which a similar treatment protocol is prescribed to all patients. VR simulations can be built from specific patient data to tailor the best treatment for an individual patient by incorporating the predictions of the simulations.

I suggest to term this concept VRID for Virtual Reality Inspired Drugs and illustrate its potential with my team's work in neurodegenerative research (Fig. 2). In this project, we are developing a 3D VR simulation named eBrain [17]. One instantiation of eBrain is inspired by reallife clinical trials in Alzheimer's patients treated with intranasally delivered insulin [18-20]. In this module, eBrain uploads patients' MRIs and simulates how a potential drug is infused into the brain through the nasal passages and diffuses into the brain tissue. It simulates how the tissue uptakes the drug and activates neurons in the substantia nigra. eBrain was employed to test hundreds of doses and compounding 


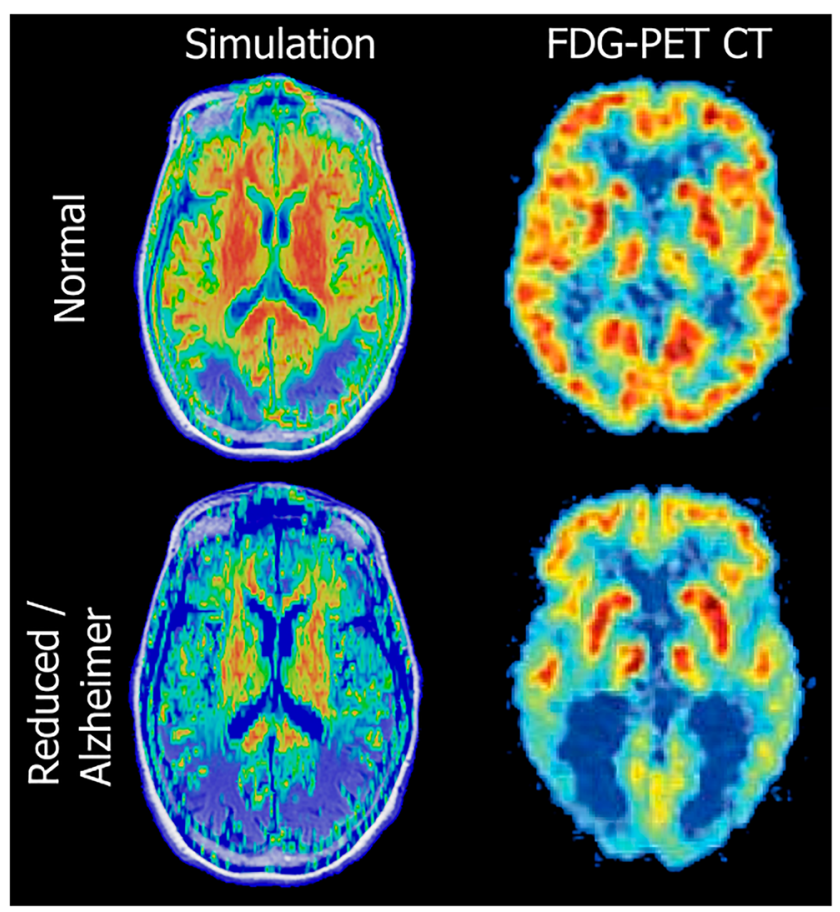

Fig. 2 VRID module vs experiment results: eBrain simulations of normal and reduced tissue uptake of patient MRI data [17] against results of FDG-PET scan of a normal and AD subjects [19]. The FDG-PET brain $\mathrm{CT}$ images are not covered by the CC BY license. Image credit to Berti et al. and S. Karger AG, Basel. All rights reserved, used with permission

treatment combinations to screen for an efficacious costeffective approach. We used this analysis to model personalized treatment in multiple scenarios. For example, eBrain modeling indicated that a combinatorial approach would help to reduce a single drug dose. This observation may lead to a reduction in drug dosing and thereby reduce the likelihood of side effects of the treatment. Currently, we are training eBrain to support more specific molecular, physiological, and pathological data. Future iterations will incorporate intracellular mechanisms to support multiple molecular pathways. Each neuron would be programed to recapitulate the intracellular and intercellular mechanisms such as protein aggregation and signal propagation. eBrain is trained to support multiple delivery systems to allow better theoretical analysis in the route of administration. Currently, eBrain supports intranasal and intracerebral delivery and we are developing simulations for physical barrier crossing delivery systems, namely intravenous, intragastric, and intrathecal. Once the physicochemical criteria are incorporated into eBrain, it will fully comply with VRID's doctrine and, in the long run, will help develop improved treatment protocols to optimize healthcare budgets and improve patient quality of life.

Acknowledgments The author wishes to thank Dr. Avi Mayo, Ben Hazan, and the members of Gateway Institute for Brain Research for their assistance. A special thanks to Dr. Charles Cohan for critically reviewing the manuscript.

\section{Compliance with Ethical Standards}

Conflict of Interest A US Patent (application \#15896344) entitled Virtual Reality Imaging of the Brain by Gateway Institute for Brain Research LLC, with the author as the inventor is filed. The material discussed in this manuscript is part of the patent.

Ethical Approval Not applicable.

Informed Consent Not applicable.

Open Access This article is distributed under the terms of the Creative Commons Attribution 4.0 International License (http:// creativecommons.org/licenses/by/4.0/), which permits unrestricted use, distribution, and reproduction in any medium, provided you give appropriate credit to the original author(s) and the source, provide a link to the Creative Commons license, and indicate if changes were made.

\section{References}

1. Mitha AP, Almekhlafi MA, Janjua MJ, Albuquerque FC, McDougall CG. Simulation and augmented reality in endovascular neurosurgery: lessons from aviation. Neurosurgery. 2013;72(Suppl 1):107-14.

2. Pallavicini F, Argenton L, Toniazzi N, Aceti L, Mantovani F. Virtual reality applications for stress management training in the military. Aerosp Med Hum Perform. 2016;87:1021-30.

3. Duncan D, et al. Using virtual reality to improve performance and user experience in manual correction of MRI segmentation errors by non-experts. J Digit Imaging. 2018.

4. Kuppersmith RB, Johnston R, Jones SB, Jenkins HA. Virtual reality surgical simulation and otolaryngology. Arch Otolaryngol Head Neck Surg. 1996;122:1297-8.

5. Colt HG, Crawford SW, Galbraith O. 3rdVirtual reality bronchoscopy simulation: a revolution in procedural training. Chest. 2001;120:1333-9.

6. Iserson KV. Ethics of virtual reality in medical education and licensure. Camb Q Healthc Ethics. 2018;27:326-32.

7. Posada-Gomez R, et al. An interactive system for fine motor rehabilitation. Rehabil Nurs. 2018;43:116-24.

8. Riva G, Wiederhold BK, Mantovani F. Neuroscience of virtual reality: from virtual exposure to embodied medicine. Cyberpsychol Behav Soc Netw. 2019;22:82-96.

9. de Rooij IJM, van de Port IGL, Visser-Meily JMA, Meijer JG. Virtual reality gait training versus non-virtual reality gait training for improving participation in subacute stroke survivors: study protocol of the ViRTAS randomized controlled trial. Trials. 2019;20: 89.

10. De Keersmaecker E, et al. Virtual reality during gait training: does it improve gait function in persons with central nervous system movement disorders? A systematic review and meta-analysis. NeuroRehabilitation. 2019;44:43-66.

11. Powell W, Simmonds MJ. Virtual reality and musculoskeletal pain: manipulating sensory cues to improve motor performance during walking. Cyberpsychol Behav Soc Netw. 2014;17:390-6.

12. Schadt EE, Sachs A, Friend S. Embracing complexity, inching closer to reality. Sci STKE 2005. In: pe40; 2005.

13. Paul SM, Mytelka DS, Dunwiddie CT, Persinger CC, Munos BH, Lindborg SR, et al. How to improve R\&D productivity: the pharmaceutical industry's grand challenge. Nat Rev Drug Discov. 2010;9:203-14. 
14. Rantanen J, Khinast J. The future of pharmaceutical manufacturing sciences. J Pharm Sci. 2015;104:3612-38.

15. Derendorf H, Meibohm B. Modeling of pharmacokinetic/ pharmacodynamic (PK/PD) relationships: concepts and perspectives. Pharm Res. 1999;16:176-85.

16. Holbein ME. Understanding FDA regulatory requirements for investigational new drug applications for sponsor-investigators. $\mathrm{J}$ Investig Med. 2009;57:688-94.

17. Setty Y. eBrain: a three dimensional simulation tool to study drug delivery in the brain. Sci Rep. 2019;9:6162.

18. Hanson LR, Frey WH. Intranasal delivery bypasses the blood-brain barrier to target therapeutic agents to the central nervous system and treat neurodegenerative disease. BMC Neurosci. 2008;9:S5.
19. Berti V, Osorio RS, Mosconi L, Li Y, de Santi S, de Leon MJ. Early detection of Alzheimer's disease with PET imaging. Neurodegener Dis. 2010;7:131-5.

20. Gupta U, et al. Intranasal drug delivery: a non-invasive approach for the better delivery of neurotherapeutics. Pharm Nanotechnol. 2017.

Publisher's Note Springer Nature remains neutral with regard to jurisdictional claims in published maps and institutional affiliations. 